\title{
Infodemia en Perú post primera ola del COVID-19: Características y factores asociados
}

\section{Infodemia in Peru after the first wave of COVID-19: Characteristics and associated factors}

\author{
https://doi.org/10.52808/bmsa.7e5.61e2.018 \\ Martin A. Vilela-Estrada ${ }^{*}$ \\ https://orcid.org/0000-0002-1494-952X \\ Ida Carbajal-Paniora ${ }^{2}$ \\ https://orcid.org/0000-0001-9764-1805 \\ Caroline B. Alcantara-Cuellar ${ }^{2}$ \\ https://orcid.org/0000-0002-8651-0487 \\ Rosario DC. Ore-Cayllahua ${ }^{2}$ \\ https://orcid.org/0000-0002-7420-7376 \\ Reyna Chura-Alanoca ${ }^{3}$ \\ https://orcid.org/0000-0001-5856-9556 \\ Erika Chumpitaz-Carrillo ${ }^{2}$ \\ https://orcid.org/0000-0002-3747-3077 \\ Victor Serna-Alarcón ${ }^{1}$ \\ https://orcid.org/0000-0002-9803-6217 \\ Lipselotte de Jesús Infante Rivera ${ }^{4}$ \\ https://orcid.org/0000-0001-6094-1070 \\ Christian R. Mejia ${ }^{3}$ \\ https://orcid.org/0000-0002-5940-7281
}

Recibido: 17/04/2021

Aceptado: 08/06/2021

\section{RESUMEN}

Una de las principales causas de deterioro de la salud mental en la pandemia fue el mal manejo de la información. El objetivo de este estudio fue evaluar la frecuencia del miedo o la percepción de mala información que transmitieron los medios de comunicación y su influencia en la salud mental de la población peruana posterior a la primera ola. Estudio transversal analítico. Que midió en casi 10 mil pobladores peruanos la percepción del miedo o exageración de las noticias con un instrumento validado previamente (Alpha de Cronbach global: 0,92), pero esto post primera ola. Se mostró las principales percepciones y se asoció al sexo, la edad y el nivel educativo. La televisión transmitía más miedo (12\% muy de acuerdo y $27 \%$ de acuerdo) y exageración (13\% muy de acuerdo y $26 \%$ de acuerdo); seguido por las redes sociales. Los hombres tuvieron más puntaje de miedo en general (Coeficiente: 1,83; IC95\%: 1,01-3,31; valor p=0,047); además, según el grado de instrucción, a comparación de los que tenían hasta primaria, los que tenían secundaria tuvieron más puntaje en total (Coeficiente: 4,53; IC95\%: 1,93-10,64; valor p=0,001), de exageración (Coeficiente: 1,73; IC95\%: 1,31-2,28; valor $\mathrm{p}<0,001$ ), de miedo (Coeficiente: 1,73; IC95\%: 1,31-2,28; valor $\mathrm{p}<0,001$ ) y de la comunicación por amigos/personal de salud (Coeficiente: 1,72; IC95\%: 1,29-2,30; valor p<0,001); ajustado por la edad y el departamento de residencia. En general, se observó que los que tenían solo educación secundaria tuvieron más miedo y fueron influenciados por los familiares y conocidos.

Palabras clave: Coronavirus, pandemia, medios de comunicación, miedo, Perú.

\begin{abstract}
One of the main causes of deterioration in mental health in the pandemic was the mishandling of information. The objective of this study was to evaluate the frequency of fear or perception of misinformation transmitted by the media and its influence on the mental health of the Peruvian population after the first wave. Analytical cross-sectional study. Which measured in almost 10,000 Peruvian inhabitants the perception of fear or exaggeration of the news with a previously validated instrument (global Cronbach's Alpha: 0.92), but this was post-first wave. The main perceptions were shown and it was associated with sex, age and educational level. Television broadcast more fear (12\% strongly agree and $27 \%$ agree) and exaggeration (13\% strongly agree and 26\% agree); followed by social media. Men had a higher fear score in general (Coefficient: 1.83; $95 \%$ CI: 1.01-3.31; $p$ value = 0.047); Furthermore, according to the level of education, compared to those who had up to primary school, those who had secondary school had a higher overall score (Coefficient: 4.53; 95\% CI: 1.93-10.64; $p$ value $=0.001$ ), of exaggeration (Coefficient: 1.73; 95\% CI: 1.31-2.28; $p$ value <0.001), of fear (Coefficient: 1.73; 95\% CI: 1.31-2.28; $p$ value <0.001) and communication by friends / health personnel (Coefficient: 1.72; 95\% CI: 1.29-2.30; $p$ value <0.001); adjusted for age and department of residence. In general, it was observed that those with only secondary education were more afraid and were influenced by relatives and acquaintances.
\end{abstract}

Key words: Coronavirus, pandemic, media, fear, Peru.

1.Escuela de Medicina Humana, Universidad Privada Antenor Orrego. Trujillo, Perú. 
*Autor de Correspondencia: mvilelae@upao.edu.pe

\section{Introducción}

La enfermedad asociada al coronavirus (SARS-COV 2) ha registrado millones de infectados en el mundo, que con el correr de los meses, provocó el colapso del sistema de salud en el Perú, así como, en otros muchos países (Escobar et al., 2020; Wang et al., 2020). El primer caso peruano se reportó el 6 de marzo del 2020, con este hecho empezó una ola de especulaciones con respecto a esta enfermedad, conocida como "infodemia", perjudicando los sistemas de salud, los medios y sus consumidores (Andreu-Sánchez \& Martín-Pascual, 2020).

Recordando que los medios de comunicación cumplen una función importante y, en el contexto de la pandemia, dicha función fue la de brindar una información fidedigna sobre la salud pública (Segura, 2020). Sin embargo, en muchas ocasiones se emitió información no verificada, que lleva a la confusión y miedo, además, del incremento de la dramatización en la narración, imágenes impactantes (como muertes del día a día) y los abordajes no científicos (de personas sin experiencia en el tema); entre los más resaltantes. Estos hechos conllevaron a un incremento de la desconfianza y el terror en la población (Arroyo-Sánchez et al., 2020).

Por lo que, el abordaje inadecuado de las noticias y hechos relacionados con la pandemia pone en mayor riesgo a la población vulnerable; evitando su adherencia a tratamientos preventivos o a estrategias de inmunización universal. Sin embargo, se sabe que la información durante la emergencia sanitaria no siempre puede ser verificada o confirmada por algún ente rector, pero su diseminación irresponsable si tiene una repercusión relevante en la población general (Depoux et al., 2020). De manera que, la "infodemia", también, repercute en la salud mental de la población, observando que esto aumenta la probabilidad de sufrir episodios de pánico, malestar y ansiedad (Shuja et al., 2020).

Diversos estudios internacionales y nacionales han demostrado la existencia de una asociación entre los medios de comunicación, los mayores niveles de ansiedad, miedo y preocupación en la población (Ahmad \& Murad, 2020; Mertens et al., 2020; Greenhawt et al., 2021). En nuestro contexto, se han realizado algunos estudios sobre la influencia de los medios de comunicación sobre el estado mental de la población peruana, pero, en muestras que se tomaron al inicio de la pandemia (Mejia et al., 2020 a; Mejia et al., 2020 b). Por lo que, el objetivo del estudio fue evaluar la frecuencia del miedo o la percepción de exageración que transmitieron los medios de comunicación y su influencia en la salud mental de la población peruana posterior a la primera ola.

\section{Materiales y Métodos}

Se realizó un estudio observacional, tipo analítico, transversal y multicéntrico. La población fue aquella que vivía en el ámbito urbano de los 24 departamentos del Perú durante los meses de diciembre del 2020 y enero del 2021. Se tomó un muestreo de tipo no aleatorio, sin embargo, se calculó la potencia estadística derivada de los cruces de las variables (siendo $80 \%$ el límite para considerarlo como adecuado, esto se logró gracias a que se tuvo más de 9 mil encuestados, por lo que se tuvo excelentes potencias). Se incluyó en la investigación a los encuestados mayores de 18 años, que expresaron estar de acuerdo con participar en la investigación, que respondiesen a las preguntas y que hayan vivido en el Perú durante los 6 meses previos a la toma de los datos. Se excluyó a aquellos que hayan dado respuestas repetidas o que no respondieron la totalidad de las preguntas; sobre todo las principales, que servían para determinar la percepción de miedo o exageración que causaron los medios de comunicación (exclusión menor al 5\% de la totalidad de las encuestas).

La variable dependiente se midió mediante el uso de una encuesta previamente validada en el Perú, en donde a través de doce preguntas se indagaba acerca de la percepción de miedo y/o de preocupación que generaban diversos medios radiales, televisivos, de redes sociales y de otras personas que pudiesen influir en cuanto a las noticias acerca del coronavirus. Cada una de las preguntas tenía una posible respuesta de percepción, en formato Likert, desde la opción muy en desacuerdo hasta la opción muy de acuerdo. Para el análisis se tuvo 4 variables dependientes: el puntaje total de las preguntas, el puntaje de la exageración, el puntaje total del miedo y el puntaje de la comunicación directa (por amistades, familia y personal de salud); el primero fue obtenido a través de la suma total de las preguntas, en cambio, las otras se obtuvieron de la suma de las 4 preguntas que generaban cada uno de esos factores (según la validación inicial) (Mejia et al., 2020 b). Se obtuvo el valor del Alpha de Cronbach global $(0,92)$, en donde se mostró un valor adecuado. Se cruzó estas versus la edad, el sexo y el grado de instrucción.

El estudio fue parte de una investigación mayor, que intentó evaluar todo el contexto de la pandemia posterior a la primera ola. La investigación basal contó con la aprobación de un comité de ética, con dicha aprobación se realizó la ejecución primaria y después se procedió a la exportación de la base a una hoja en el programa Microsoft 
Excel (para Windows). En dicha hoja se hizo el primer control de calidad, en donde se realizó la depura una a una de las variables, esto para poder detectar repeticiones o respuestas anómalas (las cuales fueron excluidas). Luego de eso se exportó esa base a una hoja en el programa Stata (versión 11,1). Es en este programa donde se realizó el análisis y su posterior extrapolación.

Para el análisis de datos se realizó una primera tabla, en donde se describió a la población según el miedo percibido, esto mediante las frecuencias y los porcentajes para las variables categóricas, así como, la media y desviación estándar para la edad. Luego, se obtuvo los coeficientes (debido a que cada una de las variables principales/dependientes fueron tomadas de manera cuantitativa), los intervalos de confianza al $95 \%$ y valores p; esto mediante el uso de los modelos lineales generalizados, con la familia Gaussian, función de enlace identity, modelos para varianza robusta y ajustados por el departamento de residencia. Es importante mencionar que todas las variables que se tomaron en los modelos bivariados fueron llevadas a los modelos multivariados, esto a pesar de que no alcancen un valor p significativo; por el hecho de saber que el sexo, la edad y el grado académicos son importantes variables y que influyen en la asociación buscada (significancia epidemiológica sobre la estadística). Además, todo se trabajó con un nivel de confianza al $95 \%$.

La ética se cuidó en todo momento, ya que, se siguió los procedimientos de la declaración de Helsinki. Además, la información recolectada fue totalmente anónima y voluntaria. Por otro lado, antes de proceder con la participación se solicitó el consentimiento verbal a cada uno de los encuestados. El proyecto basal fue aprobado por el comité de ética de la Universidad Privada Norbert Wiener (Lima-Perú).

\section{Resultados}

De los 9287 encuestados, 5066 (54,6\%) fueron mujeres, la media de edades fue de 27,0 años (desviación estándar de 11,5 años), 1538 (16,6\%) tenían hasta estudios primarios, 900 (9,7\%) de nivel secundaria, 6166 (66,4\%) estudios superiores y $683(7,3 \%)$ de posgrado. La televisión fue el principal medio de comunicación que fue percibido como el que transmitía más miedo (12,3\% muy de acuerdo y $27,4 \%$ de acuerdo) y exageración (13,3\% muy de acuerdo y $25,8 \%$ de acuerdo); seguido por las redes sociales como transmisoras de miedo (9,2\% muy de acuerdo y $22,7 \%$ de acuerdo) y exageración (10,5\% muy de acuerdo y $25,0 \%$ de acuerdo) (Tabla 1$)$.

Tabla 1. Porcentajes de percepción acerca de las noticias acerca del COVID-19 en peruanos

\begin{tabular}{|c|c|c|c|c|c|}
\hline Acerca de los medios de información acerca del coronavirus & $\begin{array}{l}\text { Muy de } \\
\text { acuerdo }\end{array}$ & $\begin{array}{c}\text { De } \\
\text { acuerdo }\end{array}$ & Indiferente & $\begin{array}{c}\text { En } \\
\text { desacuerdo }\end{array}$ & $\begin{array}{c}\text { Muy en } \\
\text { desacuerdo }\end{array}$ \\
\hline La televisión está exagerando su magnitud & $13,3 \%$ & $25,8 \%$ & $32,1 \%$ & $19,0 \%$ & $9,8 \%$ \\
\hline La televisión me genera mucho miedo & $12,3 \%$ & $27,4 \%$ & $32,4 \%$ & $19,3 \%$ & $8,6 \%$ \\
\hline Las redes sociales están exagerando su magnitud & $10,5 \%$ & $25,0 \%$ & $35,5 \%$ & $20,4 \%$ & $8,6 \%$ \\
\hline Las redes sociales me generan mucho miedo & $9,2 \%$ & $22,7 \%$ & $37,9 \%$ & $21,6 \%$ & $8,6 \%$ \\
\hline Los periódicos/diarios están exagerando su magnitud & $8,3 \%$ & $20,6 \%$ & $27,9 \%$ & $26,6 \%$ & $16,6 \%$ \\
\hline Los periódicos/diarios me generan mucho miedo & $5,4 \%$ & $17,0 \%$ & $30,3 \%$ & $28,9 \%$ & $18,4 \%$ \\
\hline La radio está exagerando su magnitud & $5,2 \%$ & $14,7 \%$ & $31,4 \%$ & $29,8 \%$ & $18,9 \%$ \\
\hline La radio me genera mucho miedo & $4,5 \%$ & $13,1 \%$ & $31,3 \%$ & $31,5 \%$ & $19,6 \%$ \\
\hline Los médicos y el personal de salud están exagerando su magnitud & $4,6 \%$ & $11,6 \%$ & $30,1 \%$ & $32,3 \%$ & $21,4 \%$ \\
\hline Los médicos y el personal de salud me generan mucho miedo & $3,7 \%$ & $10,9 \%$ & $28,4 \%$ & $33,2 \%$ & $23,8 \%$ \\
\hline Mi familia/amigos son los que están exagerando su magnitud & $4,6 \%$ & $14,4 \%$ & $31,4 \%$ & $32,0 \%$ & $17,6 \%$ \\
\hline Mi familia/amigos me generan mucho miedo & $3,0 \%$ & $8,9 \%$ & $27,7 \%$ & $35,1 \%$ & $25,3 \%$ \\
\hline
\end{tabular}

Al realizar el análisis del puntaje total de miedo o percepción de exageración se encontró que fueron los hombres los que tuvieron más puntaje en general (Coeficiente: 1,83; IC95\%: 1,01-3,31; valor p=0,047). Además, según el grado de instrucción, a comparación de los que tenían hasta primaria, los que tenían secundaria tuvieron más puntaje en total (Coeficiente: 4,53; IC95\%: 1,93-10,64; valor $\mathrm{p}=0,001$ ) y su percepción de exageración también fue relevante (Coeficiente: 1,73; IC95\%: 1,31-2,28; valor p<0,001); ajustado por la edad y el departamento de residencia (Tabla 2).

También en el análisis multivariado, a comparación de los que tenían hasta primaria, los que tenían secundaria tuvieron más puntaje de miedo (Coeficiente: 1,73; IC95\%: 1,31-2,28; valor p<0,001) y en cuanto a la percepción de inadecuada comunicación de la información por amigos/personal de salud (Coeficiente: 1,72; IC95\%: 1,29-2,30; valor $\mathrm{p}<0,001$ ); ajustado por la edad y el departamento de residencia (Tabla 3). 
Tabla 2. Modelos multivariados de la percepción total o de las preguntas de exageración en pobladores peruanos post primera ola del COVID-19 $(\mathbf{n = 9 2 7 3 )}$

\begin{tabular}{lcc}
\hline \multicolumn{1}{c}{ Variable } & Puntaje total de las preguntas & Puntaje de la exageración \\
\hline Sexo masculino & $1,83(1,01-3,31) 0,047$ & $1,06(0,84-1,35) 0,602$ \\
Edad (años)* & $0,99(0,97-1,03) 0,997$ & $1,01(0,99-1,02) 0,238$ \\
Grado académico & Esta categoría sirvió de comparación para las otras de instrucción \\
Hasta primaria & $4,53(1,93-10,64) 0,001$ & $1,73(1,31-2,28)<0,001$ \\
Secundaria & $0,79(0,32-1,96) 0,607$ & $0,97(0,73-1,28) 0,827$ \\
Universitario & $0,51(0,15-1,74) 0,280$ & $0,95(0,65-1,38) 0,778$ \\
Post grado & $\begin{array}{l}\text { el departamento de residencia. *Analizada de forma cuantitativa. } \\
\text { Los coeficientes (izquierda), los intervalos de confianza al } 95 \% \text { (dentro del paréntesis) y valores } p(\text { derecha) se }\end{array}$
\end{tabular}

Tabla 3. Modelos multivariados de la percepción del miedo o de la mala información recibida por amigos/personal de salud en pobladores peruanos post primera ola del COVID-19 (n=9273)

\begin{tabular}{lcc}
\hline \multicolumn{1}{c}{ Variable } & Puntaje total del miedo & Puntaje de la comunicación directa \\
\hline Sexo masculino & $1,06(0,84-1,35) 0,602$ & $1,18(0,97-1,44) 0,100$ \\
Edad (años)* & $1,01(0,99-1,02) 0,238$ & $1,01(0,99-1,02) 0,119$ \\
Grado académico & Esta categoría sirvió de comparación para las otras de instrucción \\
Hasta primaria & $1,73(1,31-2,28)<0,001$ & $1,72(1,29-2,30)<0,001$ \\
Secundaria & $0,97(0,73-1,28) 0,827$ & $0,97(0,71-1,32) 0,853$ \\
Universitario & $0,95(0,65-1,38) 0,778$ & $0,95(0,62-1,45) 0,809$ \\
\hline $\begin{array}{l}\text { Post grado } \\
\text { Los coeficientes (izquierda), los intervalos de confianza al 95\% (dentro del paréntesis) y valores } \mathrm{p} \\
\text { (derecha) se obtuvieron con la familia Gaussian, función de enlace identity, modelos para varianza robusta y } \\
\text { ajustados por el departamento de residencia. *Analizada de forma cuantitativa. }\end{array}$
\end{tabular}

\section{Discusión}

La televisión fue percibida aún como el medio que transmitía mayor cantidad de miedo o noticias exageradas. Esta situación se puede ejemplificar en nuestra realidad, ya que, se sabe de manera extra oficial que posterior a una noticia tendenciosa (en donde se mencionaba que la efectividad de una vacuna era muy baja, sabiendo que esta se estaba aplicando en nuestro medio), por lo que, debido a eso se paralizó las negociaciones con sus representantes (dejando desprotegidos a muchos que pudieron tener antes la inmunidad), por la gran presión social; este es un claro ejemplo de que posiblemente se tuvieron repercusiones posterior al miedo/exageración que tuvo uno de los "programas informativos" de nuestro medio. Actualmente, en un análisis semejante llevado a cabo en España, la televisión es el medio de mayor relevancia (ocupando el primer puesto, con un $81 \%$ de transmisión) y es la que genera más confianza. Paralelamente, los habitantes resaltan que la información de los medios posee tendencia a la especulación (Shaw et al., 2020). Respecto al miedo, ya se había descrito anteriormente que, en las pandemias, donde la enfermedad es poco conocida, los habitantes tienen más probabilidad de producir sentimientos de miedo y respuestas emocionales (Covello, 2003).

Es por eso lo necesario de que, en este tipo de situaciones, que puedan afectar a la salud pública, existan entes reguladores que puedan fiscalizar y hasta sancionar a los medios de comunicación (sobre todo a la televisión, que se sigue observando como el más importante en nuestra actual sociedad). Se espera que futuras investigaciones puedan mostrar si la televisión tuvo algún efecto negativo en la sociedad, en las autoridades o sus decisiones o en algún otro aspecto durante la pandemia. 
Otro medio importante fueron las redes sociales, que luego de la televisión, fue otra importante fuente de transmisión de este tipo de noticias. En ese sentido, en un estudio en Alemania se mostró que las redes sociales fueron el tercer medio de comunicación de consulta para noticias sobre COVID-19 y, se observó que las redes sociales y los sitios web estaban relacionados significativamente con el miedo a la COVID-19, mayores niveles ansiedad y depresión (Bendau et al., 2021). En una investigación de Irak se determinó que las publicaciones de las redes sociales sobre la pandemia generaban pánico por el COVID-19. Por lo que, esto provocaría un impacto negativo a nivel psicológico en las personas (Ahmad \& Murad, 2020). Es este punto más difícil de controlar, ya que, las redes sociales son libres, diversas y con regulaciones escazas en cada uno de nuestros medios. Se espera que las propias redes sociales sean las que determinen formas de controlar las noticias, se tuvo noticias de que Facebook trataba de eliminar las noticias que eran reportadas como falsas, pero no se puede llegar a precisar que tanto pudo realizarse, o incluso, en otras redes sociales (o si cuando menos tienen filtros o métodos para tratar de frenar esto). También se espera que esto sirva como línea base, para tratar de generar soluciones para lo que queda de la pandemia y otras futuras enfermedades con gran repercusión mediática.

Los hombres fueron en general los que percibieron más miedo o exageración, pero solo en el puntaje global, más no en cada uno de los otros tres puntajes individuales. Estudios previos observaron que los varones tuvieron como resultado una menor percepción de miedo y exageración con respecto a las mujeres (Mejia, Rodriguez-Alarcon, et al., 2020). De igual forma, existen reportes que muestran que las mujeres tienen mayor propensión a creer en la información no verificada y se dejan llevar por los sentimientos o emociones que pueden ser influenciados por los medios de comunicación (Pulido Acosta \& Herrera Clavero, 2015; Valdez Medina et al., 2010; The Lancet, 2020). Estos estudios nos muestran resultados opuestos a los que encontramos, sabiendo que nuestra investigación fue realizada en momentos y poblaciones distintas; respectivamente para cada una de las dos que se compararon. Por lo que, se debe seguir realizando investigación respecto a la percepción mediada por el género, ya que, esto podría estar influido por otros factores que no hemos podido cuantificar en esta investigación.

Esta investigación encontró el sólo tener educación secundaria fue un factor de riesgo para la percepción de exageración, miedo y tener más facilidad de ser influenciado por familiares y amigos. Estos hallazgos concuerdan con los obtenidos por Johnson et al, en un estudio realizado en Argentina durante la primera y segunda ola del COVID-19 en una muestra compuesta por 1410 participantes, este autor encontró que a menor nivel educativo tenían mucha mayor probabilidad de creer y diseminar noticias no verificadas o especulaciones (Johnson et al., 2020). Por otro lado, otros estudios han observado que aquellos que tienen más miedo realizan con mayor dificultad otras actividades como desempeño laboral, académico y social. En ese sentido, el estudio en 1186 estudiantes en la región de Ceuta (realizada por Pulido Acosta y Herrera Clavero) observó la relación inversa que existía entre el miedo y el rendimiento académico de los estudiantes en esta región (Pulido Acosta \& Herrera Clavero, 2015).

Una de las principales limitaciones de este estudio radicó en el método de muestreo, debido a la dificultad de poder obtener un muestreo probabilístico, el estudio tuvo la presencia del sesgo de selección, que impide la extrapolación de sus resultados a la totalidad de la población peruana. Sin embargo, debido al tamaño de la muestra y al esfuerzo del equipo de poder realizar enrolamientos proporcionales al número de habitantes de cada región participante, consideramos que las asociaciones podrían ser un aproximado de lo que sucede en la realidad. Por otro lado, debido a que se confió en la honestidad de los participantes y es difícil corroborar sus afirmaciones existe una limitación manifiesta en el sesgo de información. Sin embargo, debido a que la encuesta fue anónima y completamente personal consideramos que las afirmaciones en su mayoría son verdaderas. Por último, no se tuvo la disposición de otras muchas variables que podrían explicar lo encontrado, por lo que, se espera que futuras investigaciones generen estudios específicos para tratar de dilucidar estas relaciones, con un mejor tipo de muestreo, con diseños que permitan medir causalidad y con diversas variables (sociales, antropológicas, educativas, laborales, entre otras muchas).

Por lo tanto, se concluye que los hombres tuvieron mayor miedo y exageración percibida por los medios de comunicación. Por otro lado, se observó que los que tenían solo educación secundaria tuvieron más frecuencia en la percepción de exageración, tener miedo y ser influenciados por los familiares y conocidos. Además, la televisión se reportó como el medio que transmitía más miedo/exageración, seguida por las redes sociales.

\section{Conflicto de intereses}

Los autores declaran no tener conflicto de interés.

\section{Agradecimientos}

Los autores agradecemos a los estudiantes a nivel nacional, que apoyaron para la realización de esta investigación. Así como a la Universidad Privada Norbert Wiener, que brindó un importante soporte logístico y económico para poder lograrlo. 


\section{Referencias}

Ahmad, A. R., \& Murad, H. R. (2020). The impact of social media on panic during the COVID-19 pandemic in Iraqi Kurdistan: Online questionnaire study. Journal of Medical Internet Research, 22(5):e19556. https://doi.org/10.2196/19556

Andreu-Sánchez, C., \& Martín-Pascual, M.Á. (2020). Fake images of the SARS-CoV-2 coronavirus in the communication of information at the beginning of the first Covid-19 pandemic. El profesional de la información, 29(3):1-10. https://doi.org/10.3145/epi.2020.may.09

Arroyo-Sánchez, A. S., Cabrejo Paredes, J. E., \& Cruzado Vallejos, M. P. (2020). Infodemia, la otra pandemia durante la enfermedad por coronavirus 2019. Anales de la Facultad de Medicina, 81(2):230-233. https://doi.org/10.15381/anales.v81i2.17793

Bendau, A., Petzold, MB, Pyrkosch, L., Mascarell Maricic, L., Betzler, F., Rogoll, J., Große, J., Ströhle, A. \& Plag, J. (2021). Asociaciones entre el consumo de medios relacionados con COVID-19 y los síntomas de ansiedad, depresión y miedo relacionado con COVID-19 en la población general en Alemania. Archivos europeos de psiquiatría y neurociencia clínica , 271 (2):283-291. https://doi.org/10.1007/s00406-020-01171-6

Covello, V. T. (2003). Best practices in public health risk and crisis communication. Journal of health communication, 8(1):5-8. https://doi.org/10.1080/713851971

Depoux, A., Martin, S., Karafillakis, E., Preet, R., Wilder-Smith, A., \& Larson, H. (2020). The pandemic of social media panic travels faster than the COVID-19 outbreak. Journal of Travel Medicine, 27(3):1-2. https://doi.org/10.1093/jtm/taaa031

Escobar, G., Matta, J., Taype-Huamaní, W., Ayala, R., \& Amado, J. (2020). Características clínicoepidemiológicas de pacientes fallecidos por COVID-19 en un hospital nacional de Lima, Perú. Revista de la Facultad de Medicina Humana, 20(2):180-185. https://doi.org/10.25176/RFMH.v20i2.2940

Greenhawt, M., Kimball, S., DunnGalvin, A., Abrams, EM, Shaker, MS, Mosnaim, G., Comberiati, P., Nekliudov, NA, Blyuss, O., Teufel, M. \& Munblit, D. (2021). Influencia de los medios en la ansiedad, la utilidad de la salud y las creencias sobre la salud al comienzo del estudio de encuesta sobre la pandemia de SARS-CoV-2. Revista de medicina interna general , 36 (5):1327-1337. https://doi.org/10.1007/s11606-020-06554-y

Johnson, M. C., Saletti-Cuesta, L., \& Tumas, N. (2020). Emotions, concerns and reflections regarding the COVID-19 pandemic in Argentina. Ciencia \& saude coletiva, 25(1):2447-2456. https://doi.org/10.1590/1413$\underline{81232020256.1 .10472020}$

Mejia, C. R., Quispe-Sancho, A., Rodriguez-Alarcon, J. F., Ccasa-Valero, L., Ponce-López, V. L., Varela-Villanueva, E. S., Marticorena-Flores, R. K., Chamorro-Espinoza, S. E., Avalos-Reyes, M. S., \& Vera-Gonzales, J. J. (2020 a). Factores asociados al fatalismo ante la COVID-19 en 20 ciudades del Perú en marzo 2020. Revista Habanera de Ciencias Médicas, 19(2), 3233.

Mejia, C. R., Rodríguez-Alarcón, J. F., Carbajal, M., Pérez-Espinoza, P., Porras-Carhuamaca, L. A., Sifuentes-Rosales, J., Contreras-Cabrera, J. M., Carranza-Esteban, R. F., \& Ruiz-Mamani, P. G. (2020 b). Fatalismo ante la posibilidad de contagio por el coronavirus: Generación y validación de un instrumento (F-COVID-19). Kasmera, 48(1):1-10.https://doi.org/10.5281/zenodo.3732353

Mertens, G., Gerritsen, L., Duijndam, S., Salemink, E., \& Engelhard, I. M. (2020). Fear of the coronavirus (COVID19): Predictors in an online study conducted in March 2020. Journal of anxiety disorders, 74,:102258. https://doi.org/10.1016/j.janxdis.2020.102258

Pulido Acosta, F., \& Herrera Clavero, F. (2015). Miedo e inteligencia emocional en el contexto pluricultural de Ceuta. Anuario de Psicología/The UB Journal of Psychology, 45(2):249-263. Disponible en: https://revistes.ub.edu/index.php/Anuario-psicologia/article/view/14270/17527 (Acceso marzo 2021)

Segura, M. S. (2020). Con alerta pero sin pánico. El rol de los medios durante la pandemia. Revista de la Facultad de Ciencias Medicas (Cordoba, Argentina), 77(1): 55-58. https://doi.org/10.31053/1853.0605.v77.n1.28066

Shaw, R., Kim, Y.K. \& Hua, J. (2020). Gobernanza, tecnología y comportamiento ciudadano en una pandemia: lecciones de COVID-19 en Asia oriental. Progreso en la ciencia de desastres, 6:100090. https://doi.org/10.1016/j.pdisas.2020.100090

Shuja, K. H., Aqeel, M., Jaffar, A., \& Ahmed, A. (2020). COVID-19 Pandemic and Impending Global Mental Health Implications. Psychiatria Danubina, 32(1):32-35. https://doi.org/10.24869/psyd.2020.32 
The Lancet. (2020). COVID-19: fighting panic with information. Lancet, 395(10224):537. https://doi.org/10.1016/S0140-6736(20)30379-2

Valdez Medina, J. L., Torres Aristeo, O., Arratia López Fuen, N. I. G., \& López Romero, I. (2010). Los tipos de miedo prevalentes por generación y por sexo. Revista Electrónica de Psicología Iztacala, 13(4):163-182. https://doi.org/10.21829/azm.2020.3612274

Wang, D., Hu, B., Hu, C., Zhu, F., Liu, X., Zhang, J., Wang, B., Xiang, H., Cheng, Z., Xiong, Y., Zhao, Y., Li, Y., Wang, X. \& Peng, Z. (2020). Características clínicas de 138 pacientes hospitalizados con neumonía infectada por el nuevo coronavirus de 2019 en Wuhan, China. JAMA, 323 (11):10611069. https://doi.org/10.1001/jama.2020.1585 